\title{
A Novel Energy Aware Routing Approach using ANN Technique with Data Fusion in WSN: Review
}

\author{
Pooja Singh \\ Banasthali Vidhyapith \\ Research Scholar \\ CSE Department, Rajasthan.
}

\author{
Vikas Pareek \\ Banasthali Vidhyapith \\ AIMACT Building \\ CS Department, Rajasthan.
}

\author{
Anil K. Ahlawat \\ KIET, UPTU \\ MCA Department \\ Ghaziabad
}

\begin{abstract}
Since WSN having a limitation of the limited battery life time and if the battery will die soon i.e. Network has a finite lifespan. The most famous issue facing network designers in wireless networks is to maximize network life. In wireless communication devices, due to resource constraints sensors evidence and delivery of reliable data is a difficult task. The motivation behind this study is the some critical limitation of energy aware routing protocols. Such as; it's not easy to communicate to Base Station (BS) for that Cluster Head (CH) which is at maximum distance from Base Station therefore to make a novel mechanism by which long distance/route $\mathrm{CH}$ can be communicate with fast time with minimum energy consumptions to the base station (BS), here introduced data fusion concept. In WSN can be deployed a fusion point for accurate decision. This study includes directing the efficient use of artificial intelligent protocol with data fusion concept.
\end{abstract}

\section{General Terms}

Wireless Sensor Network, Routing, Clustering, Data fusion, Artificial Neural Network, Kohonen Self Organising Map.

\section{Keywords}

WSN, ANN, SOM, DF, Decision, Energy.

\section{INTRODUCTION}

Since wireless communications and has various benefits over basic wired network and develop low cost, low power with small sized, and revealed multifunction devices. These sensors are small in size but having the ability to detect, computing, self-organization and communication known as sensors. WSN are systems consisting of a (possibly large) number of small devices equipped with sensing capabilities, a limited amount of processing power and a radio communication chip. Usually battery powered, these units are referred to as nodes or nodes being also used in relation to the Kohonen Self Organizing maps. WSN have been adapted to a variety of applications, including: environmental, healthcare, defense, etc. In the vast majority of these applications, the WSN nodes use their sensors to gather information regarding the environment they are in, do some limited amount of processing, and forward the information to collection site. In these scenarios, two typical problems arise: 1) communicating the data through the network is a major source of power drainage, and 2) large amounts of data require a significant amount of processing power at the central site, while the processing capabilities of the nodes remain unused. The basic architecture of the sensor nodes based on its function consist of four units; Sensor, CPU, Radio and Power Unit. The first three units are responsible for the implementation of the task while the power unit is for power supply for the entire process. Function and detection module is to measure the physical and environmental conditions such as temperature through the CPU and also responsible for data processing, while the transceiver communication unit transfer data from sensor module for the user device through the base station (BS). These small sensor nodes are spread all over the region of the search for information from the surrounding, and the process, and then transferred to the base station. When considering the application area WSNs one can assume that a wired network or traditional wireless. But it's not possible because the traditional wired or wireless networks have sufficient energy resources are not limitless, and memory, and scope enough of communications and computing capabilities but on the other hand, wireless sensor network have limited resources. Although there are many similarities between the existing network and dedicated network and there are also a lot of differences and unique challenges of wireless sensor network system. As sensor network is devolution of resources so that all solutions come with the challenges facing the wireless sensor network. WSN is a high consuming power therefore it become low computing energy or power to be lead to limited battery life, by which leading to the requirements of routing protocols energy efficiency in contemplation of improve energy consumption of a node. As Besides distribution of WSN node can be viewed in a highly critical applications demand for application vary according to the nature and therefore there is a need for effective counseling protocol that should not be the only suitable for application demands, but also the help of the network with its limited resources and drives well. The motivation behind the proposed work is aware routing protocols current power to the expansion of the battery life time and high energy efficiency. Therefore, to design energy efficient WSN is first consideration. There have been many efforts to the energy awareness of WSNs, the proposed new network protocols and especially for energy conservation [1], [7]. Many researchers focused on the choice of the following strategies hops, which made hop-adjacent nodes and one or multiple connections to transfer data [8]. Many researchers have focused on identifying the date of the contract to change the way evidence between active and sleep nodes to save energy [11], [13]. Here, a neural network based on conscious energy policy mechanism with data fusion is introduced. The proposed work includes directing the efficient use of artificial intelligent protocol.

\section{LITERECTURE SURVEY: DF in WSN}

The WSN lifespan can be improvement with the data fusion support, spreading techniques and LEACH-C techniques [22]. The technique of data fusion has used to address the issues in reducing the data elements number. In the data fusion technique, collected packets of sensor data are combines into the single packet and the data packet redundancies have removed to minimize the transmission of data. Additionally, the compression of data has completed by monitoring entire 
packets' MSB (most significant bits) and then integrating the packets which have similar MSB of information. Data fusion also has the capability to transmit the data with the conception of the less energy efficiently. On the other hand some researcher has investigated the use of neural network in WSNs; [23]. For clustering KSOM neural networks have used and its examination has helped to revise the random parameters of network behaviors and the applications. Apart from these, data fusion is considered to the essential job as it is probable more to have automatic and accurate sources when compared to other protocols. Sensor prediction of data, sensor data fusion, classification of sensor data and the clustering of nodes are some of the applications of neural networks which have led to conservation of energy and the less cost of communication in WSNs. This can lead to the energy optimization routing techniques in WSN. Data aggregation or data fusion has the tendency to combine the several measurements of unreliable data to produce a more accurate signal by increasing the common signal [24]. It also has reduced the uncorrelated noise and overload of information effectively. Flat, hierarchical and the location based routing are the three major categories of routing techniques which have played a significant role to optimize the energy in WSNs. The self organizing map neural network with intelligent method has optimized the routing performance in terms of the conservation of energy and the node computation power [25]. Hierarchical routing protocols have good adaptability and the conservation of energy when compared to the plane routing protocols. Although here pointed some of the design and challenges issues in WSN. The first one is pointed that in the LEACH protocol [26]; the cluster head nodes has performed some data fusion function in which it could be transferred to the BS. These nodes are more rigorous of energy when compared to the normal nodes. Neural networks are one of the intelligent tools and it acts as the energy efficient approaches towards the wireless sensor networks. Additionally, it also has applied as a tool in entire perspectives of energy utilization reduction namely data driven, cycling of duty and mobility approaches in wireless sensor networks. Secondly pointed some of them are node deployment, dynamics of network, models of data delivery, capabilities of node, energy considerations, data aggregation/fusion, fault, and energy consumption without losing the accuracy, scalability and the transmission media. Among these, the data fusion techniques have feasible through the techniques of signal processing. Additionally, the nodes of data have the tendency to create the accurate signal by noise reduction method. The adaptive fusion of data for energy competent routing in WSN has under the data funneling with the aggregation techniques which is also based on routing methods [28]. Fusion has been executed by intermediate node with routing but the energy has been exhausted for small amount of information and it also leads to the high fusion cost. Authors pointed these strategies as the biggest disadvantages of data fusion techniques. The last third pointed that the strategy of energy-aware routing has provided the efficient usage of balanced energy in WSNs to enhance the lifespan of the network. Energy utilization was reduced by the LEACH protocol and it also has improved the lifetime of the network effectively [29]. Design and operation are highly related with the energy awareness and it also has taken into consideration for overall networks. LEACH has reduced the energy consumption by decreasing the overheads of communication between their heads of cluster and the sensors. For overcome to all these issues some researcher proposed the energy aware framework for multiple-zone data fusion technique (MZDF) in WSN. This proposed technique of
MZDF has targeted to save the efficiency of energy after performing the necessary reformation to the algorithm of conventional energy awareness to the greater dimension. Authors also have pointed that their proposed techniques has outperformed the existing LEACH approach with respect to its conservation of energy during the data fusion. MZDF has encapsulated the nodes with and without the participant in the techniques of data fusion using the energy model based on threshold.

\section{DESIGN AND IMPLEMENTATION}

In algorithms of cluster based routing effectiveness is rising the energy lifetime of wireless sensor network this study designs an energy aware routing protocol combining the data fusion and KSOM technique. The motivation of designing this system is lack of concentration of algorithms of clustering to nodes energy levels as a major parameter to formation of clusters of networks. The energy based clustering is created in this study to extend the network lifetime with enough coverage of network. The clustering based on energy generates clusters with corresponding level of energy and the utilization of energy is balanced better in complete network.

\subsection{Setup Phase of Cluster}

The protocol uses a 2 phase method of clustering Self Organizing Map followed by the algorithm of K-means [31] with a precise assessment amid the direct clustering outcomes of clustering and prototype vectors data of self organizing map. This study chooses self organizing map for clustering since it is capable to generate several input data dimensions and views the clusters into map. The dimension of input data associates to several parameters which the author requires to consider for clustering. As a preliminary phase the reason for using self organizing map is to make data pretreatment use acquired by self organizing map [32], the Kohonen self organizing map neural networks used clustering and their examination to learn random behaviours of network applications and parameters. The sensor nodes' clustering using Kohonen SOM is evaluated for different number of nodes by acquiring varied parameters of sensor nodes. KSOM is an excellent component for clustering of WSN because it is capable to lessen multi dimensional input data dimensions and view clusters into a map. Therefore the set of data is first clustered using self organizing map and then self organizing map is clustered by $\mathrm{K}$-means.

\subsection{Selection Phase of Cluster Head}

Please Various parameters are regarded in a formed cluster for choosing a cluster head. Three criterions have been regarded for selection of cluster heads namely the nearest sensor to BS [33], the sensor having highest energy level and the adjacent sensor to gravity centre of cluster. When the nearest node to base station is selected in a cluster as cluster head the author assures to use least amount of energy to spread the messages to base station. Also the adjacent sensor to cluster's gravity center assures least utilization of energy for intra cluster communication while overhead reduction of cluster head is not ensured. In case of other 2 criterions namely adjacent sensor to base station or cluster centroid the chosen cluster heads stay stable during the stage of transmission until next stage of reclustering which may precede for many rounds and it will cause quick reduction of that cluster heads while using these two criterion revealed a longer life span outcomes. After deciding the cluster base station head nodes allot proper roles to entire nodes through the proposed protocol. 


\subsection{Phase of Transmission}

After the clusters formation and choosing their associated $\mathrm{CH}$ (cluster heads) now it is the time to pass the packets of data sensed at usual nodes to their associated $\mathrm{CH}$ and after using the functions of data fusion to acquired packets by cluster heads the messages are passed to the BS (base station). The utilization of energy of entire nodes is evaluated. One of the essential data fusion problems of wireless sensor networks is the importance of utilizing an intelligent system which fuses heterogeneous information acquired from varied sources efficiently, automatically and accurately. Data fusion can reduce the data size. Even if the data had been influenced by intentional estimation or noise, the method of data fusion must be capable to identify and classify the data. Sensor data fusion is a some need of tracking applications and target detection in wireless sensor networks [34]. The consumed energy for data transmission over a distance is evaluated [35]. After every phase of transmission the author counts a new round and they also has a rotation of clusters in using criterion of maximum energy. Since their aim is to create clusters with equal levels of energy the author must have threshold for the phase of reclustering according to difference of nodes energy level. So the energy level of greater nodes of energy is verified regularly. These nodes are $\mathrm{CH}$ of the phase of last set up. The conditions are the predefined percent reduction of their energy level. When the re-clustering threshold is fulfilled the base station sends a message of re-clustering to the complete network.

\section{BASIC ARCHITECTURE OF DATA FUSION IN WSN}

In WSN data fusion architecture can be divided in mainly three types as follows:

\subsection{Centralized Architecture}

The first basic architecture of data fusion in WSN is one of the simplest architecture, where every sensor node in network senses the data and transfers the data to their central processor fusion node (central node), where processor fuses the all collected data and detected erroneous information of the data but all performance is totally depended at a single point.

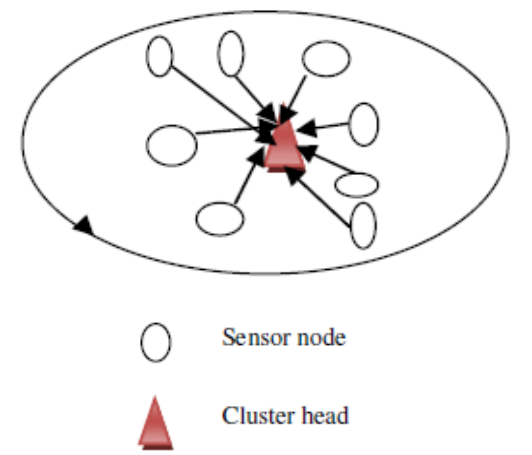

Fig 1: Centralized architecture of WSN

\subsection{Decentralized Architecture}

The second type of architecture known as decentralized architecture, in which all sensor node collect data and fused the data locally depended on the neighbouring nodes data also. Due to this phenomena there can be possible of dynamic changes in the entire network.

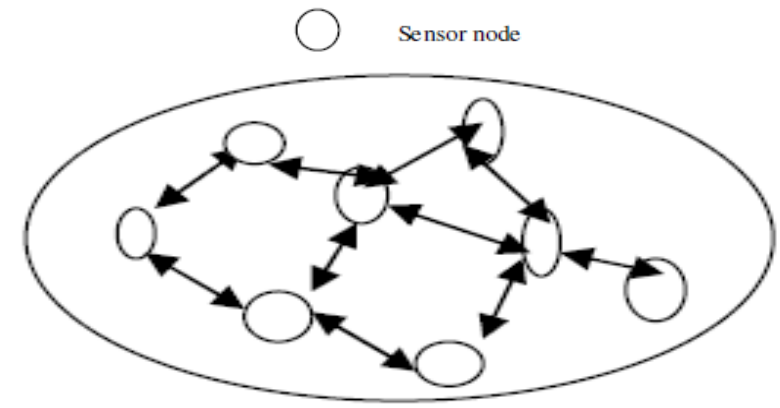

Fig 2: Decentralized architecture of WSN

\subsection{Hierarchical Architecture}

The third type of architecture is known as hierarchical architecture in which all sensor nodes are divided by the level, looking just like a tree by which the computational energy can be reduced. Here all sensor node sense the data and transfer the data to the fusion nodes by suitable routing mechanism or algorithms like directed diffusion. Due to this type of architecture the energy will be balanced among nodes in the wireless sensor network.

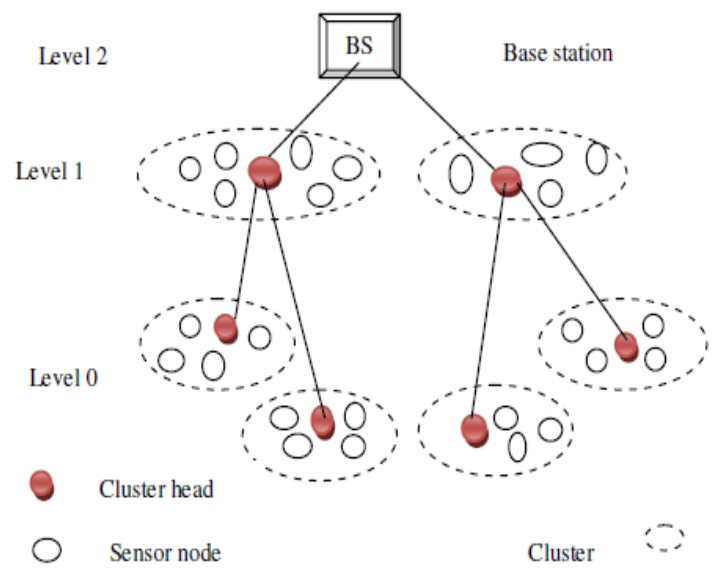

Fig 3: Hierarchical architecture of WSN.

\section{LIMITATION OF STUDY}

1. This study is constrained to wireless sensor networks.

2. This study is limited to KSOM and data fusion technique.

3. This study evaluates the intelligent energy aware routing in heterogeneous WSN environment by using Neural Network \& Data Fusion.

\section{CONCLUSION}

An intelligent energy aware routing protocol has acquired much attention in wireless sensor networks (WSN). WSN has captured the imagination and enthusiasm with applicability range. Classical routing protocols for WSNs and computational intelligence based routing protocol approach have fined tuned to make the data packets routing efficient and reliable. Low power consumptions, flexible scalability, high security, low message latency, mobility, low cost, greater availability and good fault-tolerance are some of the unique features which have associated with the wireless sensor networks. Protocols performance has considered being most promising factors in terms of efficiency of energy. Data fusion also has enhanced the QoS in the networks. This study will be 
useful for many researchers to know many factors associated with the energy aware routing protocol effectively.

\section{ACKNOWLEDGMENTS}

The authors would like to thank the anonymous reviewers for detailed comments and useful suggestion.

\section{REFERENCES}

[1] Shen C, Srisathapornphat C and Jaikaeo C (2001), Sensor information networking architecture and applications, IEEE Perspective Communication, pp 5259.

[2] Culler D, Estrin D and Srivastava M (2004), Overview of sensor networks, IEEE Computer Society, pp 41-49.

[3] Larios D F, Barbancho J, Rodriguez G, Sevillano J L, Molina F J and Leon C (2012), "Energy efficient wireless sensor network communications based on computational intelligent data fusion for environmental monitoring," IET Communications, vol. 6, no. 14, pp. 2189-2197.

[4] Li, Xiao-Hui, and Zhi-Hong Guan, "energy aware routing in WSN Using local betweenness centrality", International Journal of Distributed Sensor Networks Volume 2013 (2013), Article ID 307038, pages 9. 2013.

[5] Mainwaring A, Culler D, Polastre J, Szewczyk R and Anderson J (2002), Wireless Sensor networks for habitat monitoring, in WSNA.

[6] Shah R and Rabaey J (2002), "Energy aware routing for low energy adhoc sensor networks," in Proceedings of the IEEE Wireless Communications and Networking Conference (IEEE WCNC '02), pp. 350-355, Orlando, Fla, USA.

[7] $\mathrm{XH} \mathrm{Li,} \mathrm{SH} \mathrm{Hong,} \mathrm{KL} \mathrm{Fang;} \mathrm{"Location-based} \mathrm{self-}$ adaptive routing algorithm for wireless sensor networks in home automation", EURASIP Journal on Embedded Systems, 2011 - Springer, open access research article.

[8] Li X H, Hong S H, and Fang K (2011), "A greedy and heuristic routing algorithm for wireless sensor networks in home automation," IET Communications, vol. 5, no. 13, pp. 1797-1805.

[9] M. Umadevi and M. Devapriya Open Access An Enhanced Ant Colony Based Approach to Optimize the Usage of Critical Node in Wireless Sensor Networks", Graph algorithms, high performance Implementation and its applications (ICGHIA 2014), procedia computer science; volume 47, 2015, pages 452-459

[10] Bisnik N, Abouzeid A and Isler V (2006), Stochastic event capture using mobile sensors subject to a quality metric, In MobiCom.

[11] Cui G and Mohapatra P (2004), Power conservation and quality of surveillance in target tracking sensor networks, In MobiCom.

[12] Chair Z and Varshney P (2000), Optimal data fusion in multiple sensor detection systems, IEEE Trans Acrosp. Electron. System, 22 (1)

[13] Enami N, Askari Moghadam R, Haghighat A (2010), A survey on application of neural networks in Energy Conservation of wireless sensor networks, In Recent trends in wireless and mobile networks WIMO, 2010 Proceedings, Ankara, Turkey, pp 283-294.

[14] Kulakov A, Davcev D and Trajkovski G (2005), Application of wavelet neural networks in wireless sensor networks, $6^{\text {th }}$ International Conference on Software Engineering, Artificial Intelligence, Networking and Parallel/Distributed Computing and First ACIS International Workshop on Self Assembling Wireless Networks, p 262-267.

[15] Aslam N, Philips W, Robertson W and Siva Kumar S H (2010), A multi criterion optimization technique for energy efficient cluster formation in wireless sensor networks, In Information Fusion, Elsevier.

[16] Anatasi G, Conti M and Pasarrella A (2009), Energy conservation in wireless sensor networks a survey, In Adhoc Networks, Volume 7, Issue 3, Elsevier, pp 537568 .

[17] Liu M, Cao J, Chen G and Wang X (2009), An EnergyAware Routing Protocol in Wireless Sensor Networks, Sensors, Vol-9, pp 445-462.

[18] Baranidharan B and Shanthi B (2010), A Survey on Energy Efficient Protocols for Wireless Sensor Networks, International Journal of Computer Applications, 11(10).

[19] Enami N, Moghadam A R, Dadashtabar K and Hoseini M (2010), Neural Network Based Energy Efficiency in Wireless Sensor Networks: A Survey, International Journal of Computer Science and Engineering Survey, $1(1)$.

[20] Singh K S, Singh P M and Singh K D (2010), Routing Protocols in Wireless Sensor Networks- A Survey, International Journal of Computer Science and Engineering Survey, 1(2)

[21] Padmanabhan, K., and Kamalakkannan, P., (2011), "A Study on Energy Efficient Routing Protocols in Wireless Sensor Networks", European Journal of Scientific Research, Vol. 60, No. 4, pp. 499-511.

[22] Nagarajan M and Geetha T (2012), Wireless Sensor Network's Life Time Enhancement With Aid Of Data Fusion, Leach-C And Spreading Techniques, I J I T E , 3(1-2), pp 375-380

[23] Nimbalkar K J (2012), Use of Neural Networks in WSNs: A Survey, International Journal of advancement in electronics and computer engineering, 1(3), pp 93-98.

[24] Nivetha G (2012), Energy Optimization Routing Techniques in Wireless Sensor Networks, International Journal of Advanced Research in Computer Science and Software Engineering, 2(7).

[25] Amri S and Kaddachi L M (2014), SOM Based EnergyEfficient Multi-hop Hierarchical Routing Protocol for Wireless Networks, NNGT Int. J. of. Artificial Intelligence, Vol-1.

[26] Kashani A, Mosavian I and Mahriyar H (2014), A Method for Reduction of Energy Consumption in Wireless Sensor Networks with using Neural Networks, Indian Journal of Fundamental and Applied Life Sciences, 4(S3), pp 1043-1050.

[27] Mondal K R and Sarddar D (2014), Data-Centric Routing Protocols in Wireless Sensor Networks: A 
Survey, COMPUSOFT: An International Journal of Advanced Computer Technology, 3(2).

[28] Kumar A S and Illango P (2015), Data Funnelling in Wireless Sensor Networks: A Comparative Study, Indian Journal of Science and Technology, 8(5), pp 472-480.

[29] Gurbani P, Acharya H and Jain A. (2016), Hierarchical Cluster Based Energy Efficient Routing Protocol for Wireless Sensor Networks: A Survey, International Journal of Computer Science and Information Technologies, 7(2), pp 682-687.

[30] Yadav S G S and Chitra A (2016), MZDF: An Energy Aware Framework for Multi-Zone Data Fusion Technique in WSN, International Journal of Applied Engineering Research, 11(4), pp 2263-2270.

[31] Vesanto J, Alhoniemi E (2000), Clustering of Self Organizing Map. In: IEEE Transactions on Neural Networks, Vol. 11, No. 3, 2000, pp. 586-600.

[32] Dehni L, Kief F, Bennani Y (2005), Power Control and Clustering in Wireless Sensor Networks. In: Proceedings of Med-Hoc-Net: Mediterranean Ad Hoc Networking Workshop, France

[33] Dehni L, Krief F, Bennani Y (2005). Power Control and Clustering in Wireless Sensor Networks. In: Challenges in Ad Hoc Networking, vol , p.31-4

[34] Smith, D., Singh, S. (2006) "Approaches to Multisensor Data Fusion in Target Tracking: A Survey", IEEE TRANSACTIONS ON KNOWLEDGE AND DATA ENGINEERING, Vol. 18, No. 12, pp.

[35] Heinzelman W, Chandrakasan A, Balakrishnan $H$. (2000), Energy-Efficient Communication Protocol for Wireless Microsensor Networks. In: Proc. $33^{\text {rd }}$ Hawaii Int'1. Conf.Sys. Sc.

[36] Saber AMRI and Med Lassaad KADDACHI. (2014), SOM based energy efficient multi hop hierarchical routing protocol for wsn, NNGT Int. J. of Artifical Intellegent, vol. 1, July 2014.

\section{AUTHOR PROFILE}

Pooja Singh is pursuing $\mathrm{PhD}$ from Banastahli Vidyapith University and she has more than 8 year of teaching experience. She received the B.Tech(I.T) degree from purvanchal university \& M.Tech (C.S.E) degree from banasthali vidhyapith university. Currently working as Assistant Professor since 2008 with the Department of Computer Science and Information Technology, with the Amity School of Engineering and Technology, New Delhi.

Dr Vikas Pareek did his P.hD \& integrated M.Tech from Banasthali University, Rajsthan. He received the B.E degree from Engineering College of Bikaner; University of Rajsthan.
He is Associate Professor with banasthali vidhyapith, Rajsthan.

Dr Anil K Ahlawat did his PhD from IP university, he received the M.Tech (C.S.E) degree from Kurukshetra university \& M.Phil in Instrumentation with Gold Medal from IIT Roorkee . He is a HoD \& Professor with the Department of MCA, with the Krishna Institute of Engineering and Technology, Ghaziabad.

\section{APPENDIX}

Table Appendix - Classification of neural network based on energy conservation methods.

\begin{tabular}{|c|c|c|c|}
\hline $\begin{array}{l}\text { Sr. } \\
\text { No. }\end{array}$ & $\begin{array}{l}\text { ANN Concept } \\
\text { Role }\end{array}$ & $\begin{array}{l}\text { ANN } \\
\text { TOPOLOGY } \\
\text { APPLIED }\end{array}$ & $\begin{array}{l}\text { ENERGY } \\
\text { SAVING } \\
\text { CLASS }\end{array}$ \\
\hline 1 & $\begin{array}{l}\text { Energy Efficient } \\
\text { Path Discovery }\end{array}$ & SOM, BP & $\begin{array}{l}\text { Duty Cycling } \\
\text { Routing }\end{array}$ \\
\hline 2 & $\begin{array}{l}\text { Energy Efficient } \\
\text { Nodes Clustering }\end{array}$ & SOM & $\begin{array}{l}\text { Duty Cycling } \\
\text { Cluster Based } \\
\text { Routing }\end{array}$ \\
\hline 3 & $\begin{array}{l}\text { Cluster Head } \\
\text { Selection }\end{array}$ & SOM & $\begin{array}{l}\text { Duty Cycling } \\
\text { Cluster Based } \\
\text { Routing }\end{array}$ \\
\hline 4 & $\begin{array}{l}\text { Data } \\
\text { Aggregation / } \\
\text { Fusion }\end{array}$ & SOM, BP & $\begin{array}{l}\text { Reduction of } \\
\text { Data in network } \\
\text { Processing }\end{array}$ \\
\hline 5 & Data Association & Hopfield & $\begin{array}{l}\text { Reduction of } \\
\text { Data in network } \\
\text { Processing }\end{array}$ \\
\hline 6 & $\begin{array}{l}\text { Mobile Data } \\
\text { Association }\end{array}$ & $\begin{array}{l}\text { Competitive } \\
\text { Hopfield }\end{array}$ & $\begin{array}{l}\text { Reduction of } \\
\text { Data in network } \\
\text { Processing }\end{array}$ \\
\hline 7 & $\begin{array}{l}\text { Context / Data } \\
\text { Classification }\end{array}$ & $\begin{array}{l}\text { SOM, MEMs } \\
\text { \& ARTI }\end{array}$ & $\begin{array}{l}\text { Reduction of } \\
\text { Data in network } \\
\text { Processing }\end{array}$ \\
\hline 8 & Data Prediction & $\begin{array}{l}\text { BP, Elman, } \\
\text { ARMA \& } \\
\text { RBF }\end{array}$ & $\begin{array}{l}\text { Data Reduction } \\
\text { - Data } \\
\text { Prediction \& } \\
\text { Duty Cycling }\end{array}$ \\
\hline
\end{tabular}

